\title{
Transanal endoscopic microsurgery for early rectal cancer: single center experience
}

\author{
Narimantas Samalavicius ${ }^{1,2}$, Marijus Ambrazevicius ${ }^{1}$, Alfredas Kilius $^{1}$, Kestutis Petrulis ${ }^{1}$ \\ ${ }^{1}$ Center of Oncosurgery, Institute of Oncology, Vilnius University, Vilnius, Lithuania \\ ${ }^{2}$ Clinic of Internal Diseases, Family Medicine and Oncology of Medical Faculty, Vilnius University, Vilnius, Lithuania
}

Videosurgery Miniinv 2014; 9 (4): 603-607

DOI: $10.5114 /$ wiitm.2014.44138

\begin{abstract}
Introduction: The use of transanal endoscopic microsurgery (TEM) is increasing due to the ability to perform minimally invasive local treatment with large full-thickness local excision under improved vision.

Aim: To evaluate the initial experience with TEM for early rectal cancer in a single center.

Material and methods: From February 2010 to November 2013 a total of 20 patients underwent TEM for early rectal cancer. Nine were women and 11 men, age range 39 to 88 years (median: 71 years). The postoperative surveillance protocol, which includes rigid proctoscopy, carcinoembryonic antigen (CEA) and endorectal ultrasound every 3 months during the first 2 years, was applied to all patients after TEM.

Results: Final histology revealed 14 (70\%) lesions to be T1 and 6 (30\%) T2 cancers. There were no postoperative complications. All 6 patients in the pT2 group and those in the PT1 group with unfavorable histology were offered adjuvant chemoradiotherapy or immediate radical surgery. Patients were followed up from 2 to 35 months (median: 21 months). There was one local recurrence (5\%) in a patient who refused to undergo abdominoperineal excision for $T 1$ low rectal cancer, had unfavorable histology after TEM, and for which reason underwent postoperative chemoradiation. The patient had abdominoperineal resection 7 months after TEM (rpT2NOMO). One patient was lost to follow-up. The rest of the patients are alive and disease-free.

Conclusions: In our hands, TEM was an alternative to total mesorectal excision in patients with low-risk early rectal cancer. Further follow-up is necessary to evaluate recurrence and survival rates after TEM for patients with invasive rectal cancer.
\end{abstract}

Key words: transanal endoscopic microsurgery, early rectal cancer, recurrence, survival.

\section{Introduction}

Transanal endoscopic microsurgery (TEM) was devised in an animal model by Buess et al. in 1983 as an alternative to transanal excision (TAE) [1]. The use of TEM is increasing due to the ability to perform minimally invasive local treatment with large full-thickness local excision under improved vision. It has a well-established role in removal of rectal adenomas [2-4], but its purpose in local treatment of early rectal cancer is yet to be defined. The TEM is comparable to radical resection in terms of recurrence but with far less morbidity and mortality in favorable T1 tumors [5-7]. The requirement of costly equipment and difficult tumor selection remain the major drawbacks of this method, although they have to be weighed against improved functional outcomes and quality of life compared to radical resection $[6,8]$.

\section{Address for correspondence}

Marijus Ambrazevicius MD, Institute of Oncology Vilnius University, Santariskiu 1, LT-08660 Vilnius, Lithuania, phone: +37061832201,

e-mail: ambrazevicius.m@gmail.com 


\section{Aim}

The aim of this study was to evaluate single center experience with TEM for early rectal cancer (defined as T1-T2 rectal cancer without any nodal involvement (NO)).

\section{Material and methods}

From 19.02.2010 to 26.11.2013 a total of $20 \mathrm{pa}$ tients (Table I) underwent TEM for early rectal cancer. Nine were women and 11 men, age range 39 to 88 years (median: 71 years). Rectal lesions were from $10 \mathrm{~mm}$ to $30 \mathrm{~mm}$ in diameter (median $23.5 \mathrm{~mm}$ ). Nine (45\%) tumors were located in the lower third of the rectum, 10 (50\%) in the middle third and $1(5 \%)$ in the upper third. All patients underwent pelvic magnetic resonance imaging (MRI) and endorectal ultrasound, as well as abdominal ultrasound and chest X-ray preoperatively.

All patients who underwent rectal adenocarcinoma were offered TEM as an alternative to open total mesorectal excision (TME). Selection criteria for TEM were well or moderately differentiated T1 rectal cancer without any nodal involvement (NO) and no histological signs of poor prognosis on preoperative biopsy. Two patients (an 88-year-old patient (case no. 7) and an 82-year-old patient (case no. 16)) were found to have T2N1 rectal cancer on $M R I$ - due to refusal of radical resection and presence of significant co-morbidities TEM was performed.

All operations were performed under general anesthesia, in lithotomy, prone jack-knife, left lateral or right lateral position (depending on the exact location of the tumor). Standard TEM equipment was used. Full thickness excision with $1 \mathrm{~cm}$ safety margin was attempted, followed by closing of the rectal wall defect in one-layer running monocryl 3-0 suture using silver clips. In one case (TEM was performed for $\mathrm{T} 2$ rectal cancer), the abdominal cavity was penetrated and two-layer closure was preferred.

One patient (case no. 10) with pT1 rectal cancer had co-existing stage II G3 peripheral lung cancer, which was treated by lower left lobectomy and chemoradiotherapy in 2010.

The postoperative surveillance protocol, which includes rigid proctoscopy, carcinoembryonic antigen (CEA) and endorectal ultrasound every 3 months during the first 2 years, was applied to all patients after TEM.

\section{Results}

Final histology revealed 14 (70\%) lesions to be $\mathrm{T} 1$ and 6 (30\%) T2 cancers. Five (25\%) tumors were well differentiated (G1), and the remaining 15 (75\%) were moderately differentiated (G2). In all 20 cases resection margins were adequate and disease-free. Operative time ranged from 30 to 300 min (median: $72.5 \mathrm{~min}$ ). Median length of stay was 4 days (range: 2-15 days).

Postoperative recovery was uneventful in all cases. One patient in the PT1 group (case no. 2) underwent TEM for an upper 1/3 polyp which was understaged preoperatively as carcinoma in situ, and open partial TME was proposed and performed; no residual tumor or positive lymph nodes were detected postoperatively. In 2 cases from the pT1 group (cases no. 6 and no. 8) lymphovascular invasion was present on final pathology - both patients were sent for postoperative adjuvant chemoradiotherapy (long course radiotherapy with 5 FU based chemotherapy). One of them (case no. 6) was lost to follow-up.

All 6 patients in the pT2 group were offered adjuvant chemoradiotherapy or immediate radical surgery. An 88-year-old man (case no. 7) refused any other therapy or surveillance. Three patients (cases no. $1,3,5)$ were treated with adjuvant chemoradiotherapy. Cases no. 13 and 16 underwent immediate abdominoperineal resection - no residual tumor or positive lymph nodes were detected postoperatively.

Patients were followed up from 2 to 35 months (median: 21 months). One patient (case no. 6) was lost to follow-up. There was one local recurrence (5\%) in a patient (case no. 8) who refused to undergo abdominoperineal excision for T1 low rectal cancer, had unfavorable histology after TEM, and for which reason underwent postoperative chemoradiation. The patient underwent abdominoperineal resection 7 months after TEM (rpT2NOMO). The rest of the patients are alive and disease-free.

\section{Discussion}

The management of early rectal cancer aims to offer a cure while minimizing the morbidity and mortality of the treatment. Total mesorectal excision is a gold standard today for rectal cancer treatment in terms of local control, prevention of distal spread and long-term survival, but may result in permanent stoma and a significant chance of sexual or urinary dysfunction (up to 40\%), anastomotic leakage (5-10\%) 


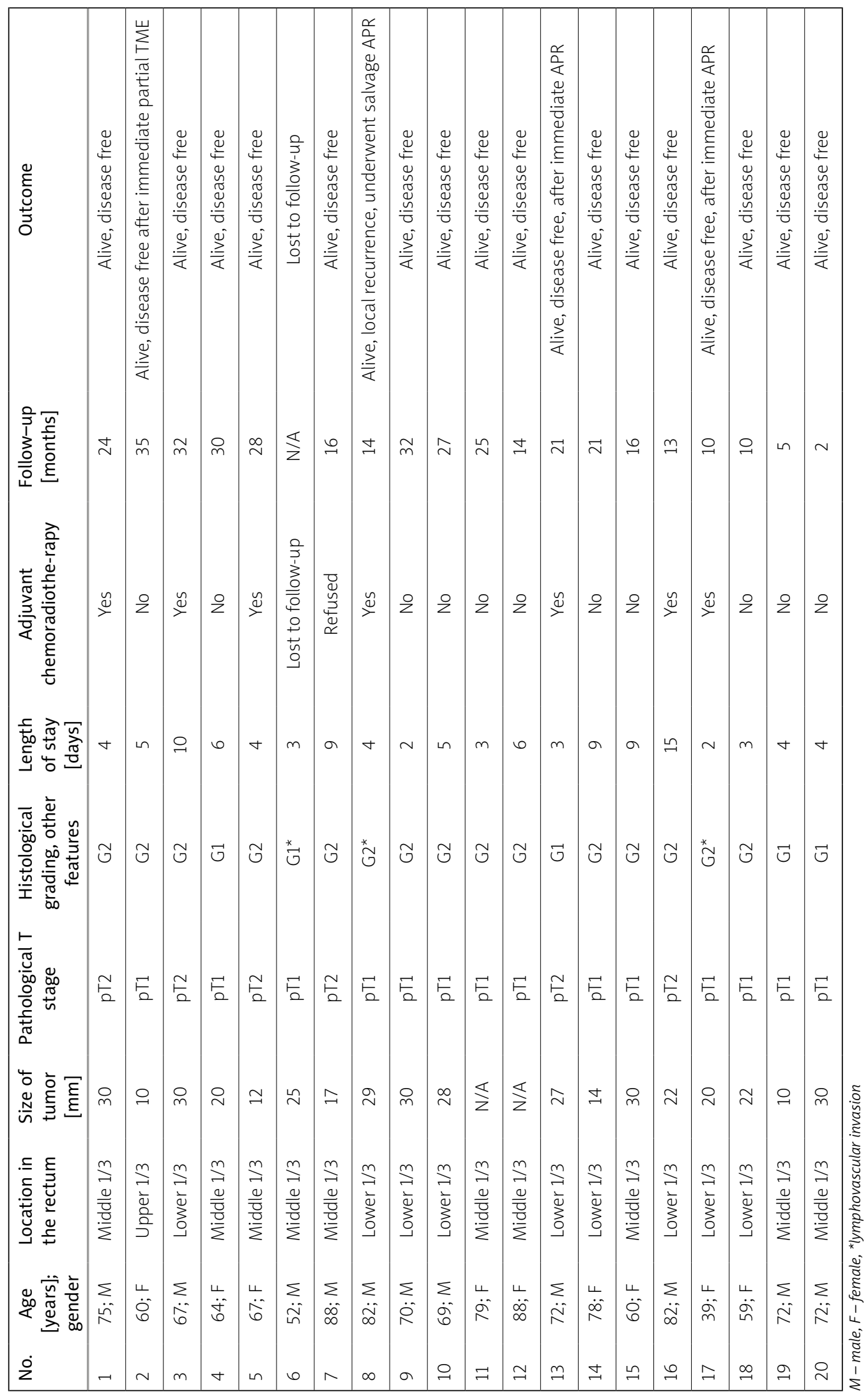


and long-term functional bowel disturbance [9]. The TEM may offer the opportunity of cure with less detriment.

Standard TEM equipment was used in our series. However, to overcome high costs, a 'glove port' and standard laparoscopic instruments can be utilized [10, 11]. When performing TEM, determination of the peritoneal reflection is important to avoid injury to the bowel. However, the location and length of the peritoneal reflection are highly variable [12].

There was one perforation of the abdominal cavity in our study, which was closed with double layer suture. No complications were detected afterwards - this is in agreement with Morino et al. [13], who in their recent study reported no influence of peritoneal perforation during TEM short-term or oncologic outcomes.

The successful treatment of rectal carcinoma by TEM depends on careful patient selection. Correct staging by imaging is crucial to define patient eligibility: MRI is useful to assess the nodal disease [14], whereas endorectal ultrasound scan has a sensitivity of $>80 \%$ and a specificity of $>90 \%$ for T-staging [15]. Nevertheless, endorectal ultrasound is a very user-dependent method and can result in inaccurately staged rectal cancer (up to $44.8 \%$ of tumors as reported in a study based on the UK TEM database [16]).

Accepted low-risk criteria of $\mathrm{T} 1$ rectal carcinoma suitable for local treatment are well or moderately differentiated lesion, slight carcinoma invasion of the muscularis mucosa (sm1), smaller than $3 \mathrm{~cm}$ in diameter, less than $40 \%$ wall circumference and no sign of lymphovascular invasion [5, 17, 18]. In a study based on an international multicentre TEM registry, Bach et al. [19] defined three histopathological variables, which independently predict local recurrence-free survival: depth of tumor invasion (a composite of pT and Sm category), maximum tumor diameter and presence of intramural lymphovascular invasion.

The surgical margin status has a significant influence on the success of treatment: $R 1, R x, R \leq 1 \mathrm{~mm}$ or high-risk $\mathrm{T} 1$ rectal tumors increase the local recurrence rate from $6 \%$ to $39 \%$. However, immediate radical surgery after non-radical local excision of rectal pT1 carcinoma reduces the recurrence rate to $6 \%$ [20].

As for transanal excision in pT2 lesions, local therapy alone is related to high risk of local recurrence: overall recurrence rates (including patients who did and did not have chemoradiation) range from $6 \%$ to $18 \%$ [21]. Our strategy in the current series was not to leave a single patient without further treatment if pT2 cancer was found in the final histology.

Lezoche et al. [22] compared endoluminal locoregional resection by TEM to laparoscopic TME for T2 rectal cancer after chemoradiotherapy. They concluded that the probability of developing recurrence or metastases and cancer-related survival rate were similar in both groups. However, short-term results significantly favored TEM in terms of operating time, stoma rate, blood loss and transfusions, need for analgesia and hospital stay.

Ongoing trials (local excision versus TME in downstaged T2/T3 low rectal cancer after radiochemotherapy; neoadjuvant chemoradiation and local excision for uT2uN0) will help to answer the question concerning the treatment of higher rectal cancer stages by the local technique after neoadjuvant therapy [23].

\section{Conclusions}

In our hands, TEM was an alternative to standard total mesorectal excision in patients with low-risk early rectal cancer. Further follow-up is necessary to evaluate recurrence and survival rates after TEM for patients with invasive rectal cancer.

\section{References}

1. Buess G, Theiss R, Hutterer F, et al. [Transanal endoscopic surgery of the rectum - testing a new method in animal experiments]. Leber Magen Darm 1983; 13: 73-7.

2. Middleton PF, Sutherland LM, Maddern GJ. Transanal endoscopic microsurgery. A systematic review. Dis Colon Rectum 2005; 48: 270-84.

3. De Graaf EJR, Burger JWA, Van ljsseldijk AL, et al. Transanal endoscopic microsurgery is superior to transanal excision of rectal adenomas. Colorectal Dis 2011; 13: 762-7.

4. Samalavicius NE, Kilius A, Petrulis K, et al. Transanal endoscopic microsurgery: experience with first 50 cases. Acta Medica Lituanica 2012; 19: 45-9.

5. Suppiah A, Maslekar S, Alabi A, et al. Transanal endoscopic microsurgery in early rectal cancer: time for a trial? Colorectal Dis 2008; 10: 314-27.

6. Winde G, Nottberg H, Keller R, et al. Surgical cure for early rectal carcinomas (T1). Transanal endoscopic microsurgery vs. anterior resection. Dis Colon Rectum 1996; 39: 969-76.

7. Lezoche E, Guerrieri M, Paganini AM, et al. Transanal endoscopic versus total mesorectal laparoscopic resections of T2-NO low rectal cancers after neoadjuvant treatment: a prospective randomized trial with a 3-years minimum follow-up period. Surg Endosc 2005; 19: 751-6. 
8. Cataldo PA, O’Brien S, Osler T. Transanal endoscopic microsurgery: a prospective evaluation of functional results. Dis Colon Rectum 2005; 48: 1366-71.

9. Nastro P, Beral D, Hartley J, et al. Local excision of rectal cancer: review of literature. Dig Surg 2005; 22: 6-15.

10. Alessandro C, Daniela M, Michele M, et al. Glove port technique for transanal endoscopic microsurgery. Int I Surg Oncol 2012; 2012: 1-4.

11. Hompes R, Ris F, Cunningham C, et al. Transanal glove port is a safe and cost-effective alternative for transanal endoscopic microsurgery. Br J Surg 2012; 99: 1429-35.

12. Kenig J, Richter P. Definition of the rectum and level of the peritoneal reflection - still a matter of debate. Videosurgery Miniinv 2013; 8: 183-6.

13. Morino M, Allaix ME, Famiglietti F, et al. Does peritoneal perforation affect short- and long-term outcomes after transanal endoscopic microsurgery? Surg Endosc 2013; 27: 181-8.

14. Muthusamy VR, Chang KJ. Optimal methods for staging rectal cancer. Clin Cancer Res 2007; 13: 6877s-84s.

15. Puli SR, Bechtold ML, Reddy JBK, et al. How good is endoscopic ultrasound in differentiating various T stages of rectal cancer? Meta-analysis and systematic review. Ann Surg Oncol 2009; 16: 254-65.

16. Ashraf S, Hompes R, Slater A, et al. A critical appraisal of endorectal ultrasound and transanal endoscopic microsurgery and decision-making in early rectal cancer. Colorectal Dis 2012; 14: 821-6.

17. Nascimbeni R, Burgart LJ, Nivatvongs S, et al. Risk of lymph node metastasis in $T 1$ carcinoma of the colon and rectum. Dis Colon Rectum 2002; 45: 200-6.

18. Kikuchi R, Takano M, Takagi K, et al. Management of early invasive colorectal cancer. Risk of recurrence and clinical guidelines. Dis Colon Rectum 1995; 38: 1286-95.

19. Bach SP, Hill J, Monson JRT, et al. A predictive model for local recurrence after transanal endoscopic microsurgery for rectal cancer. Br J Surg 2009; 96: 280-90.

20. Borschitz T, Heintz A, Junginger T. The influence of histopathologic criteria on the long-term prognosis of locally excised pT1 rectal carcinomas: results of local excision (transanal endoscopic microsurgery) and immediate reoperation. Dis Colon Rectum 2006; 49: 1492-506.

21. Kunitake H, Abbas MA. Transanal endoscopic microsurgery for rectal tumors: a review. The Permanente Journal 2012; 16: 45.

22. Lezoche E, Baldarelli M, Lezoche G, et al. Randomized clinical trial of endoluminal locoregional resection versus laparoscopic total mesorectal excision for 72 rectal cancer after neoadjuvant therapy. Br J Surg 2012; 99: 1211-8.

23. Léonard D, Colin JF, Remue C, et al. Transanal endoscopic microsurgery: long-term experience, indication expansion, and technical improvements. Surg Endosc 2012; 26: 312-22.

Received: 24.01.2014, accepted: 2.04.2014. 